\title{
Phonon Spectra of Small Colloidal II-VI Semiconductor Nanocrystals
}

\author{
Volodymyr Dzhagan, ${ }^{1}$ Mykhailo Valakh,, ${ }^{1}$ Nikolai Mel'nik, ${ }^{2}$ Olexandra Rayevska, ${ }^{3}$ \\ Irina Lokteva, ${ }^{4}$ Joanna Kolny-Olesiak, ${ }^{5}$ and Dietrich R. T. Zahn ${ }^{6}$ \\ ${ }^{1}$ V. Ye. Lashkaryov Institute of Semiconductor Physics, The National Academy Sciences of Ukraine, Prospekt Nauky 45, \\ Kyiv 03028, Ukraine \\ ${ }^{2}$ P. N. Lebedev Physical Institute, Russian Academy of Sciences, Leninsky Prosp. 53, Moscow 119991, Russia \\ ${ }^{3}$ L.V. Pysarzhevskiy Institute of Physical Chemistry, National Academy Sciences of Ukraine, Prospekt Nauky 31, Kyiv 03028, Ukraine \\ ${ }^{4}$ Nanomaterials for Optoelectronics Group, Department of Materials Science, University of Erlangen-Nüremberg, \\ Martensstrße 791058 Erlangen, Germany \\ ${ }^{5}$ Energy and Semiconductor Research Laboratory, Department of Physics, \\ Carl von Ossietzky University of Oldenburg, Carl-von-Ossietzky Strße 9-11, 26111 Oldenburg, Germany \\ ${ }^{6}$ Semiconductor Physics, Chemnitz University of Technology, Reichenhainer Strße 70, 09107 Chemnitz, Germany
}

Correspondence should be addressed to Volodymyr Dzhagan, dzhagan@isp.kiev.ua

Received 6 April 2011; Accepted 24 June 2011

Academic Editor: Sandip Dhara

Copyright ( $) 2012$ Volodymyr Dzhagan et al. This is an open access article distributed under the Creative Commons Attribution License, which permits unrestricted use, distribution, and reproduction in any medium, provided the original work is properly cited.

\begin{abstract}
Resonant Raman spectroscopy has been employed to explore the first- and higher-order phonon spectra of several kinds of IIVI nanocrystals (NCs), with the aim of better understanding of the nature of phonon modes and forming a unified view onto the vibrational spectrum of semiconductor NCs. Particularly, besides the previously discussed TO, SO, LO, and 2LO modes, the combinational modes of $\mathrm{TO}+\mathrm{LO}$ and $\mathrm{SO}+\mathrm{LO}$ can be assumed to account for the lineshape of the spectrum below $2 \mathrm{LO}$ band. No trace of $2 \mathrm{TO}$ or $2 \mathrm{SO}$ band was detected, what can be the result of the dominance of Fröhlich mechanism in electron-phonon coupling in II-VI compounds. The resonant phonon Raman spectrum of NCs smaller than $2 \mathrm{~nm}$ is shown to be dominated by a broad feature similar to the SO mode of larger NCs or phonon density of states of a bulk crystal.
\end{abstract}

The understanding of the phonon spectrum of semiconductor nanocrystals (NCs), electron-phonon coupling (EPC), dependence of the phonon spectra on the NC size, surfacebound moieties, and extended environment is of a large fundamental and application significance [1-11] due to the determinant role phonons play in the optical and electrical properties of semiconductor nanostructures $[12,13]$. Though a number of in-depth studies have addressed the phonon spectra of colloidal [14-20], glass-embedded [2123] NCs, and epitaxial nanostructures [24-26], a significant divergence of the results exists concerning both the nature of the modes and their response to such factors as size, surface conditions, properties of the hosting medium and so forth. In particular, the apparently similar Raman spectra of a wide range of compounds (e.g., II-VI, III-V, and IV) and NC morphologies (dots, rods, tetrapods, wires, and discs) have been assigned differently.

Recently, attempts were made to build a general picture of the phonon spectrum of various semiconductor NCs, based on analysis of the experimental Raman data and appropriate models [14, 27, 28]. This paper further explores the first- and higher-order phonon spectra of several kinds of II-VI NCs, as well as their similarity to other compounds, with the aim of better understanding of the vibrational spectrum of small NCs. High-quality NC samples were selected for this study in order to resolve Raman features, which are usually smeared by size dispersion, structural disorder, or low signal-to-noise ratio.

The NC samples studied in this work have been prepared by means of colloidal chemistry according to protocols 


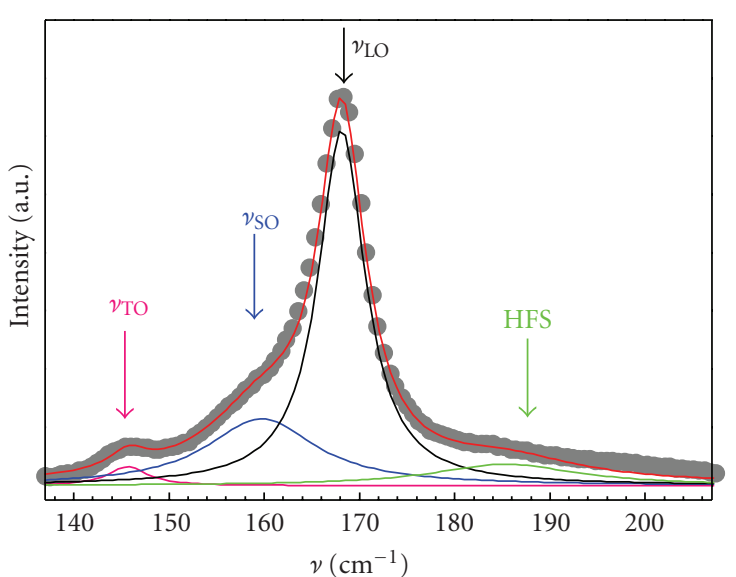

(a)



(b)

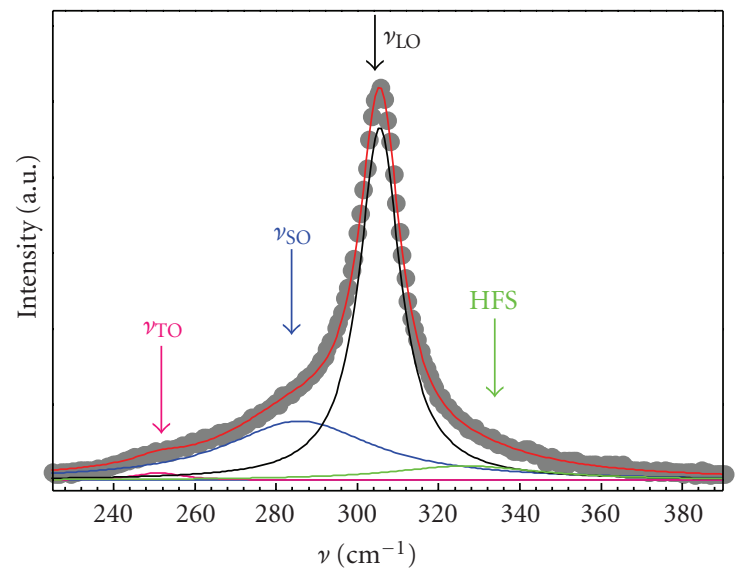

(c)

Figure 1: First-order Raman spectra of CdTe (a), CdSe (b), and CdS (c) NCs in the range of LO phonon with multi-Lorentzian fitting. Indicated is the assignment of the phonon modes.

reported elsewhere [29-32], and CdSe, CdS, and CdTe NC samples of the same mean diameter $(\sim 4 \mathrm{~nm})$ were selected. Another sort of NCs studied were ultrasmall NCs of about $1.8 \mathrm{~nm}$ mean diameter, which revealed distinct Raman spectra. The attempts to measure the size and shape of these NCs directly by electron microscopy were unsuccessful [19], obviously due to ultrasmall NC size and charging of the stabilizing polymer. The broad X-ray features of these ultrasmall NCs [3] indicated their small size or/and noticeable structural deformations due to dominance of the surface atoms. Therefore, the size of the latter ultrasmall NCs has been estimated from the energy of the first excitonic maximum [19].

The main features observed in resonant Raman scattering spectra of II-VI NCs are the longitudinal optical (LO) phonon mode (fundamental) and its overtones (Figures 1 and 2), which are shifted to lower frequencies and broadened compared to the bulk crystal. The nature of a low-frequency shoulder of the LO peak, commonly observed for different kinds of semiconductor NCs, is still under discussion. It is related either with phonons at $k \neq 0$ [33], surface optical (SO) [23], zone-edge (ZE) [22] phonons, or quantized optical phonons (vibrons) with a quantum number $n>1$ $[17,34,35]$. Fitting the Raman spectrum in the range of LO mode with two Lorentzians, corresponding to SO (or ZE) and LO phonon or to vibrons with different quantum numbers, is widely applicable for II-VI NCs $[15,18,23$, 36]. However, the similar shoulder has been observed in bulk alloys [37]. The alloys, even defect free, are known to possess a kind of disorder caused by the fluctuation of the electrostatic potential due to the inhomogeneous spatial distribution of the counterpart ions. This kind of disorder, along with the structural disorder, can lead to activation of some vibrational modes (disorder-activated ZE phonons) [37]. Investigation of the surface-related (SO) vibrations in NCs is important not only due to its potential in probing the NC-environment interaction [4], but also because they may take part in the optical transitions in NCs [38].

In a majority of experimental studies, the low-frequency shoulder being not resolved as a separate peak may lead to large errors in determination of its frequency position and widths. In addition, the LO phonon peak possesses in many cases a noticeable high-frequency shoulder (HFS, Figures $1(\mathrm{a})$ and $1(\mathrm{~b}))$, which probably originates from vibrations 




(a)



(b)

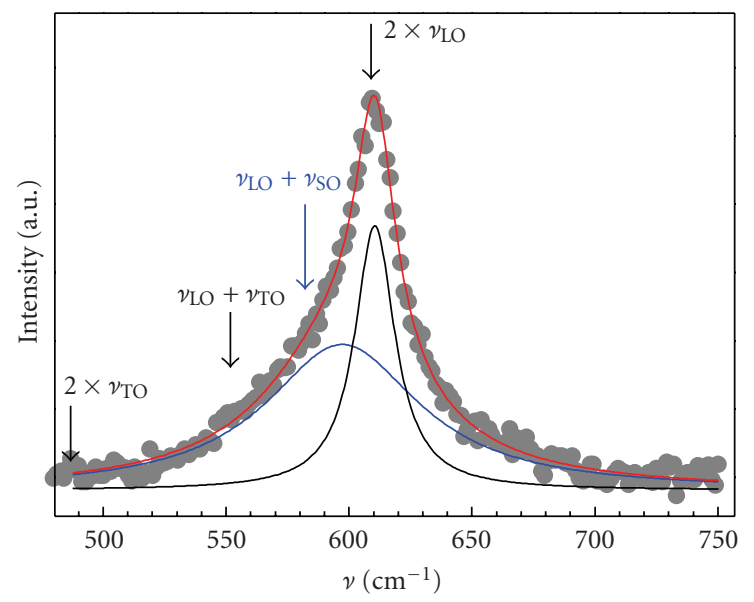

(c)

Figure 2: Multi-Lorentzian fitting Raman spectra of CdTe (a), CdSe (b), and CdS (c) NCs in the range of 2LO phonon. The assignment of the phonon modes is indicated.

of tellurium (selenium) on the CdTe (CdSe) NC surface or coupled optical-acoustical phonon modes [28, 29, 32]. Trying to satisfactory fit the whole spectrum in the range of the LO peak without taking into account the HFS can lead to overestimation of the frequency and bandwidth of the SO mode and affect the width of the LO peak and its ratio to overtones.

Our recent study of high-quality and monodispersed CdTe NCs revealed that the fitting of the lower-frequency asymmetry of the LO peak requires at least two components, attributed to TO and SO modes, correspondingly [28]. Here we extend this approach to fitting of the RRS spectra of the first-order spectra of CdSe and CdS NCs (Figure 1), as well as their overtones (Figure 2). According to this approach [28], the lowest frequency component, peaked at $145 \mathrm{~cm}^{-1}$ for CdTe NCs, $177 \mathrm{~cm}^{-1}$ for CdSe NCs, and $250 \mathrm{~cm}^{-1}$ for CdS NCs, may be assigned to TO mode, because of its frequency proximity to the frequencies for the corresponding bulk crystals. The broad component between this mode and LO may be naturally assigned to the SO mode(s), as predicted by theory [39]. Quantitatively, the SO mode frequency is calculated using the same formalism as in previous works $[18,23,31] — \approx 200 \mathrm{~cm}^{-1}$ for CdSe NCs, $\approx 280 \mathrm{~cm}^{-1}$ for CdS, and $\approx 160 \mathrm{~cm}^{-1}$ for CdTe NCs-being in good correlation with the position of the component marked as "SO" in our experimental spectra in Figure 1, as well as with other reports $[18,23,31]$.

The general appearance of the first-order resonant Raman spectrum of II-VI NCs studied here (Figure 1) is well reproduced by the model of quantized optical vibrations of spherical NC $[34,35]$, with the $(l=0, n=1)$ vibron corresponding to (fundamental) LO mode. However, as the experimental $\nu_{\text {SO }}$ does not vary noticeably with NC size [32], it is unlikely to be related with the $\left(l_{p}=2, n=1\right)$ vibron mode revealing a strong size dependence of its frequency $[34,35]$. Instead, the experimentally observed SO mode may be related to the density of $l_{p}=2$ vibron modes, centered around $180 \mathrm{~cm}^{-1}$ for CdSe and $240-255 \mathrm{~cm}^{-1}$ for CdS [34].

Based on a simple qualitative argument of the SO mode intensity, $I_{\mathrm{SO}}$, being proportional to the NC surface-tovolume $(S / V)$ ratio, the $I_{\mathrm{SO}} / I_{\mathrm{LO}}$ is expected to increase with NC size decrease. However, this approach may be 




Figure 3: Resonant Raman scattering spectra of a series of CdSe NCs of different size, measured with $\lambda_{\text {exc }}=458 \mathrm{~nm}$ at $50 \mathrm{~K}$.

oversimplified and prone to be offset by other factors, for example, by the saturation of the $\mathrm{SO}$ vibration intensity below a certain NC size at which the SO mode field extends over the whole NC volume. As a result, no noticeable dependence of $I_{\mathrm{SO}} / I_{\mathrm{LO}}$ on NC size will take place below certain critical NC diameter, as can be seen in Figure 3 for NC diameter $d$ decreasing from 4 down to $2 \mathrm{~nm}$, with the corresponding $S / V$ increasing almost twice (from about 35 up to $60 \%$ ). Furthermore, the analysis of the literature shows that the relative intensity of the SO mode can be very close for different NC morphologies and dimensions (dots, rods, tetrapods, and wires) with various $S / V$.

The nonsystematic sensitivity of the SO peak parameters to capping of the CdSe core with a ZnS (or CdS) shell [40] introduces further ambiguity in understanding of the nature of this mode. Based on similarity of the $\mathrm{SO}$ mode parameters for different NC morphologies, as well as existence of similar low-frequency asymmetry for bulk alloys [37]; one may assume it to be induced by the presence of atoms with coordination differing from the rest of (bulk) atoms. In NCs, these are the surface atoms with undercoordinated bonds, while in alloys these can be the atoms adjacent to the "impurity" atom or a structural defect. This kind of disorder can lead to activation in Raman scattering of vibrational modes forbidden for perfect crystal, particularly ZE phonons [22].

The ZE phonons, that is, phonons from the boundary of the Brillouin zone, where TO and LO branches approach each other, are forbidden in Raman spectra due to their large $k$ (compared to that of the probing light). However, they can be activated in Raman by a certain degree of disorder in the lattice. The surface of the NC is a case of such a disorder, with the spatial dimension of 1-2 lattice constants-the same length where ZE phonons exist. The probability of the appearance of the surface-related modes in Raman spectrum may be higher in smaller NCs not only due to a larger surface-to-volume ratio but also due to a larger overlap of the carrier's wavefunction with surface. As to the role of the surface-trapped excitons, it may be not relevant, because

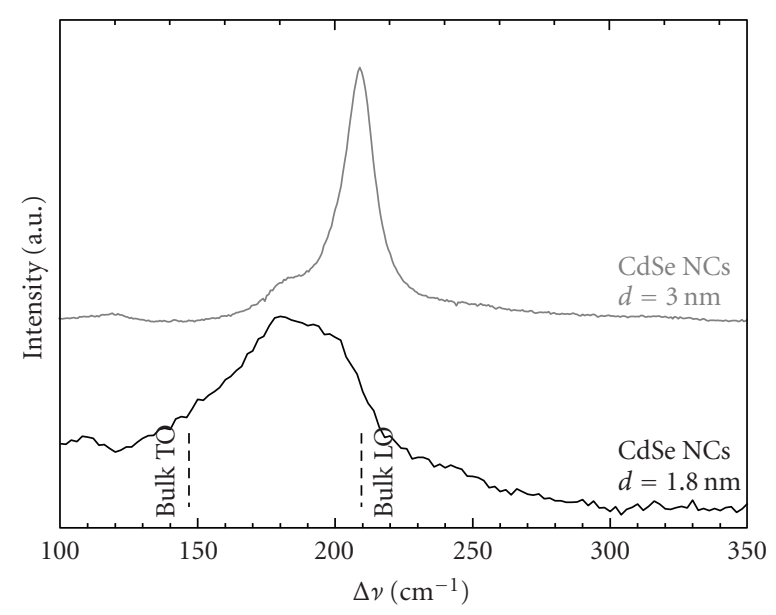

Figure 4: Resonant Raman spectrum of ultrasmall CdSe NCs $(d \approx$ $1.8 \mathrm{~nm}$ ), measured with $\lambda_{\text {exc }}=442 \mathrm{~nm}$.

the trapping times are usually much longer than the duration of the Raman scattering event.

Though the low-frequency shoulders of the LO overtones resemble strongly that of the fundamental peak, the fitting of the spectra shows that the former cannot be attributed to the nSO modes. The frequency position of the shoulder is much larger than the corresponding $n \times \nu_{\mathrm{SO}}$. More viable is the assignment of these shoulders to the $\mathrm{SO}+\mathrm{nLO}(n=$ 1,2 ) sum modes (Figure 2). At least the frequency position fits well to such a combination. This assignment can reveal new aspects of coupling between the strongly confined optical phonons in small semiconductor NC. Nevertheless, the contribution of second-order scattering on ZE phonons cannot be excluded and needs further studying. The secondorder spectrum is also featured by the high-frequency shoulder centered around $20-40 \mathrm{~cm}^{-1}$ above the $2 \mathrm{LO}$ band (Figure 2), similar to the HFS in the first-order spectrum (Figure 1).

The structure of the Raman spectrum has been observed to change qualitatively for $\mathrm{NC}$ smaller than $2 \mathrm{~nm}$. The firstorder spectrum of CdSe NCs as small as $1.8 \mathrm{~nm}$ (Figure 4) reveals a broad feature between bulk TO and LO frequencies, thus in the region of the SO mode observed for larger NCs. Simple estimation shows that at $d$ as small as $1.8 \mathrm{~nm}$, most of the atoms can be classified as surface ones and such NCs can therefore be an illustration of a limiting case of small NC which do not support the bulk-like LO (and TO) phonon modes. Their spectrum more likely resembles the phonon density of states (DOS) predicted for bulk CdSe and CdSe nanowires with a diameter of $d=2 \mathrm{~nm}$ [41] or the spectrum of molecular-like clusters $[16,42]$. From the viewpoint of the model based on bulk dispersion curves, the strong spatial confinement along with lattice distortion due to the reconstruction induced by a domination of surface atoms can lead to the situation when only phonons (TO and LO) close to (bulk) zone boundary exist in NCs. These ZE phonons manifest themselves in Raman spectrum as a single broad feature because of a very small TO-LO splitting near 
the zone boundary, as has been inferred previously for the description of IR phonon spectra of $\mathrm{Cd}_{56} \mathrm{Te}_{32}$ clusters [43].

In conclusion, the resonant Raman spectra of several kinds of II-VI NCs have been analyzed. It is shown that similar set of phonon modes can be derived from fitting the spectra of high enough quality. Particularly, besides the previously discussed TO, SO, and LO modes, the combinational modes of $\mathrm{TO}+\mathrm{LO}$ and $\mathrm{SO}+\mathrm{LO}$ can be assumed to account for the lineshape of the spectrum below 2LO band. No trace of $2 \mathrm{TO}$ or $2 \mathrm{SO}$ band was detected, what can be the result of the dominant Fröhlich mechanism in electron-phonon coupling in II-VI compounds. The resonant phonon Raman spectrum of NCs smaller than $2 \mathrm{~nm}$ is shown to be dominated by a broad feature similar to the SO mode of larger NCs or a DOS of a bulk crystal.

\section{Acknowledgments}

The work was supported by NAS of Ukraine (32-08-10Ukr), Russian Foundation for Basic Research (10-02-90468Ukr), Ukrainian State Fund for Fundamental Researches (Ф40.2/068, 41.1/017), and Alexander von Humboldt Foundation (Research Group Linkage Programme).

\section{References}

[1] G. Gouadec and P. Colomban, "Raman spectroscopy of nanostructures and nanosized materials," Journal of Raman Spectroscopy, vol. 38, no. 6, pp. 598-603, 2007.

[2] F. García-Santamaría, S. Brovelli, R. Viswanatha et al., "Breakdown of volume scaling in auger recombination in CdSe/CdS heteronanocrystals: the role of the core-shell interface," Nano Letters, vol. 11, no. 2, pp. 687-693, 2011.

[3] Q. H. Zhong, "Optical phonon modes and electron-phonon interaction in a quantized CdS spherical film," Physica B, vol. 404, no. 8-11, pp. 1241-1246, 2009.

[4] P. M. Chassaing, F. Demangeot, V. Paillard et al., "Surface optical phonons as a probe of organic ligands on $\mathrm{ZnO}$ nanoparticles: an investigation using a dielectric continuum model and Raman spectrometry," Physical Review B, vol. 77, no. 15, Article ID 153306, 2008.

[5] C. Chen, M. Dutta, and M. A. Stroscio, "Surface-optical phonon assisted transitions in quantum dots," Journal of Applied Physics, vol. 96, no. 4, Article ID 2049, pp. 2049-2054, 2004.

[6] S. K. Gupta, S. Sahoo, P. K. Jha, A. K. Arora, and Y. M. Azhniuk, "Observation of torsional mode in $\mathrm{CdS}_{1-x}$ Sex nanoparticles in a borosilicate glass," Journal of Applied Physics, vol. 106, no. 2, Article ID 024307, 2009.

[7] M. P. Lisitsa, M. Y. Valakh, and N. K. Konovets, "Phonons in mixed CdxZn1-xS semiconductors," Physica Status Solidi B, vol. 34, pp. 269-278, 1969.

[8] S. Biswas, M. Dutta, P. Snee, and M. A. Stroscio, "Phonon modes in semiconductor quantum dots," Chinese Journal of Physics, vol. 49, no. 1, pp. 92-99, 2011.

[9] S. Ma, R. Livingstone, B. Zhao, and J. R. Lombardi, "Enhanced raman spectroscopy of nanostructured semiconductor phonon modes," Journal of Physical Chemistry Letters, vol. 2, no. 6, pp. 671-674, 2011.

[10] T. T. K. Chi, G. Gouadec, P. Colomban, G. Wang, L. Mazerolles, and N. Q. Liem, "Off-resonance Raman analysis of wurtzite CdS ground to the nanoscale: structural and size-related effects," Journal of Raman Spectroscopy, vol. 42, no. 5, pp. 1007-1015, 2011.

[11] P. Kusch, H. Lange, M. Artemyev, and C. Thomsen, "Sizedependence of the anharmonicities in the vibrational potential of colloidal CdSe nanocrystals," Solid State Communications, vol. 151, no. 1, pp. 67-70, 2011.

[12] S. V. Kilina, D. S. Kilin, and O. V. Prezhdo, "Breaking the phonon bottleneck in PbSe and CdSe quantum dots: timedomain density functional theory of charge carrier relaxation," ACS Nano, vol. 3, no. 1, pp. 93-99, 2009.

[13] V. I. Klimov, "Linear and nonlinear optical spectroscopy of semiconductor nanocrystals," in Handbook of Nanostructured Materials and Nanotechnology, H. S. Nalwa, Ed., vol. 4 of Optical Properties, pp. 473-481, Academic Press, New York, NY, USA, 2000.

[14] M. I. Vasilevskiy and C. Trallero-Giner, "Resonant Raman scattering in spherical quantum dots: II-VI versus III-V semiconductor nanocrystals," Physica Status Solidi (B), vol. 247, no. 6, pp. 1488-1491, 2010.

[15] D. S. Liang, L. Shen, Z. B. Wang, Y. P. Cui, J. Y. Zhang, and Y. H. Ye, "Evolution of luminescence with shell's thickness in colloidal CdSe/CdS," Chinese Physics Letters, vol. 25, no. 12, pp. 4431-4434, 2008.

[16] Y. S. Park, A. Dmytruk, I. Dmitruk et al., "Aqueous phase synthesized CdSe nanoparticles with well-defined numbers of constituent atoms," Journal of Physical Chemistry C, vol. 114, no. 44, pp. 18834-18840, 2010.

[17] A. G. Rolo and M. I. Vasilevskiy, "Raman spectroscopy of optical phonons confined in semiconductor quantum dots and nanocrystals," Journal of Raman Spectroscopy, vol. 38, no. 6, pp. 618-633, 2007.

[18] H. Lange, M. Mohr, M. Artemyev, U. Woggon, T. Niermann, and C. Thomsen, "Optical phonons in colloidal CdSe nanorods," Physica Status Solidi (B), vol. 247, no. 10, pp. 24882497, 2010.

[19] O. E. Rayevska, G. Y. Grodzyuk, V. M. Dzhagan et al., "Synthesis and characterization of white-emitting CdS quantum dots stabilized with polyethylenimine," Journal of Physical Chemistry C, vol. 114, pp. 22478-22486, 2010.

[20] V. Dzhagan, O. Rayevska, O. Stroyuk et al., "Resonant Raman spectroscopy of confined and surface phonons in CdSe-caped CdS nanocrystals," Physica Status Solidi C, vol. 6, no. 2, pp. 2043-2046, 2009.

[21] Y. M. Azhniuk, V. V. Lopushansky, A. V. Gomonnai, V. O. Yukhymchuk, I. I. Turok, and Y. I. Studenyak, "Spectroscopic studies of thermal treatment effect on the composition and size of $\mathrm{CdS}_{1-x} \mathrm{Se}_{x}$ nanocrystals in borosilicate glass," Journal of Physics and Chemistry of Solids, vol. 69, no. 1, pp. 139-146, 2008.

[22] A. Ingale and K. C. Rustagi, "Raman spectra of semiconductor nanoparticles: disorder-activated phonons," Physical Review B, vol. 58, no. 11, pp. 7197-7204, 1998.

[23] A. Roy and A. K. Sood, "Surface and confined optical phonons in $\mathrm{CdS}_{x}-\mathrm{Se}_{1-x}$ nanoparticles in a glass matrix," Physical Review $B$, vol. 53, no. 18, pp. 12127-12132, 1996.

[24] A. G. Milekhin, A. I. Toropov, A. K. Bakarov et al., "Interface phonons in InAs and AlAs quantum dot structures," Physical Review B, vol. 70, no. 8, Article ID 085314, 5 pages, 2004.

[25] A. I. Mashin, A. V. Nezhdanov, D. O. Filatov et al., "Confocal Raman microscopy of self-assembled GeSi/Si(001) Islands," Semiconductors, vol. 44, no. 11, pp. 1504-1510, 2010.

[26] A. M. Yaremko, V. O. Yukhymchuk, M. Y. Valakh et al., "Theoretical and experimental investigations of single- and multilayer structures with SiGe nanoislands," Materials Science and Engineering C, vol. 23, no. 6-8, pp. 1027-1031, 2003. 
[27] C. C. Yang and S. Li, "Size-dependent raman red shifts of semiconductor nanocrystals," Journal of Physical Chemistry B, vol. 112, no. 45, pp. 14193-14197, 2008.

[28] V. Dzhagan, M. Y. Valakh, J. Kolny-Olesiak et al., "Resonant Raman study of phonons in high-quality colloidal CdTe nanocrystals," Applied Physics Letters, vol. 94, no. 24, Article ID 243101, 2009.

[29] V. Dzhagan, I. Lokteva, C. Himcinschi et al., "Phonon Raman spectra of colloidal CdTe nanocrystals: effect of size, nonstoichiometry and ligand exchange," Nanoscale Research Letters, vol. 6, no. 1, pp. 79-88, 2011.

[30] A. E. Raevskaya, A. L. Stroyuk, S. Y. Kuchmii et al., "Optical study of CdS- and ZnS-passivated CdSe nanocrystals in gelatin films," Journal of Physics, vol. 19, no. 38, Article ID 386237, 2007.

[31] V. M. Dzhagan, M. Y. Valakh, A. E. Raevskaya et al., "Size effects on Raman spectra of small CdSe nanocrystals in polymer films," Nanotechnology, vol. 19, Article ID 305707, 2007.

[32] V. M. Dzhagan, I. Lokteva, M. Y. Valakh et al., "Spectral features above LO phonon frequency in resonant Raman scattering spectra of small CdSe nanocrystals," Journal of Applied Physics, vol. 106, Article ID 084318, 2009.

[33] H. Richter, Z. P. Wang, and L. Ley, "The one phonon Raman spectrum in microcrystalline silicon," Solid State Communications, vol. 39, no. 5, pp. 625-629, 1981.

[34] A. G. Rolo, M. I. Vasilevskiy, M. Hamma, and C. TralleroGiner, "Anomalous first-order Raman scattering in III-V quantum dots: optical deformation potential interaction," Physical Review B, vol. 78, no. 8, Article ID 081304, 2008.

[35] M. P. Chamberlain, C. Trallero-Giner, and M. Cardona, "Theory of one-phonon Raman scattering in semiconductor microcrystallites," Physical Review B, vol. 51, no. 3, pp. 16801693, 1995.

[36] L. Lu, X. L. Xu, W. T. Liang, and H. F. Lu, "Raman analysis of CdSe/CdS core-shell quantum dots with different CdS shell thickness," Journal of Physics Condensed Matter, vol. 19, no. 40, Article ID 406221, 2007.

[37] D. Nesheva, Z. Aneva, M. J. ŠĆepanović et al., "Composition and structure of $\mathrm{Zn}_{x} \mathrm{Cd}_{1-x}$ Se single layers prepared by thermal evaporation of ZnSe and CdSe," Journal of Physics: Conference Series, vol. 253, no. 1, 2010.

[38] C. Chen, M. Dutta, and M. A. Stroscio, "Surface-optical phonon assisted transitions in quantum dots," Journal of Applied Physics, vol. 96, no. 4, Article ID 2049, pp. 2049-2054, 2004.

[39] S. Hayashi and R. Ruppin, "Raman scattering from GaP microcrystals: analysis of the surface phonon peak," Journal of Physics C, vol. 18, no. 12, Article ID 2583, 1985.

[40] V. M. Dzhagan, M. Y. Valakh, O. E. Raevska et al., "The influence of shell parameters on phonons in core-shell nanocrystals: a resonant Raman study," Nanotechnology, vol. 20, Article ID 365704, 2009.

[41] M. Mohr and C. Thomsen, "Phonons in bulk CdSe and CdSe nanowires," Nanotechnology, vol. 20, no. 11, Article ID 115707, 2009.

[42] S. Xu, C. Wang, and Y. Cui, "Theoretical investigation of CdSe clusters: influence of solvent and ligand on nanocrystals," Journal of Molecular Modeling, vol. 16, no. 3, pp. 469-473, 2010.

[43] A. G. Rolo, M. I. Vasilevskiy, N. P. Gaponik, A. L. Rogach, and M. J. M. Gomes, "Confined optical vibrations in CdTe quantum dots and clusters," Physica Status Solidi (B), vol. 229, no. 1, pp. 433-437, 2002. 


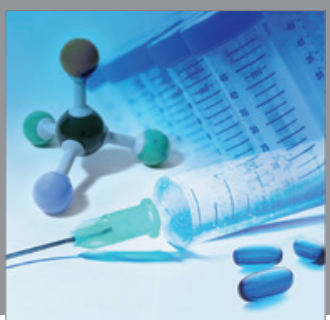

International Journal of

Medicinal Chemistry

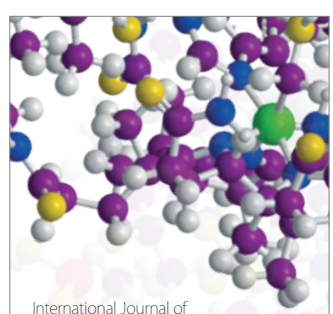

Carbohydrate Chemistry

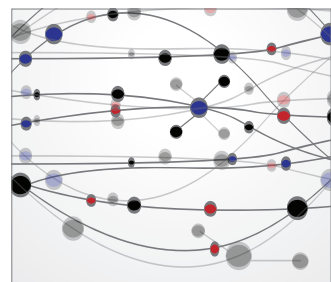

The Scientific World Journal
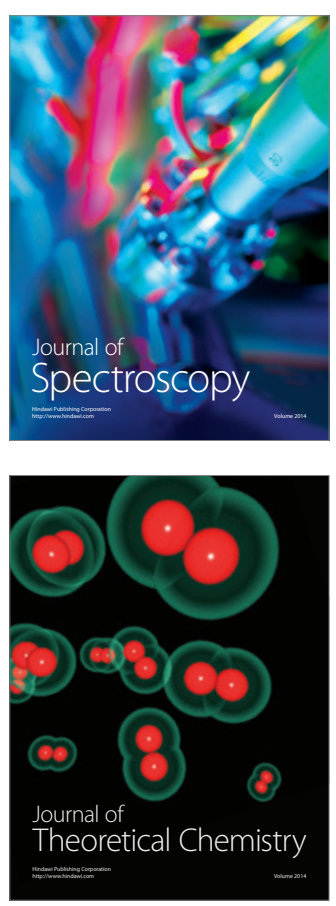


Submit your manuscripts at

http://www.hindawi.com
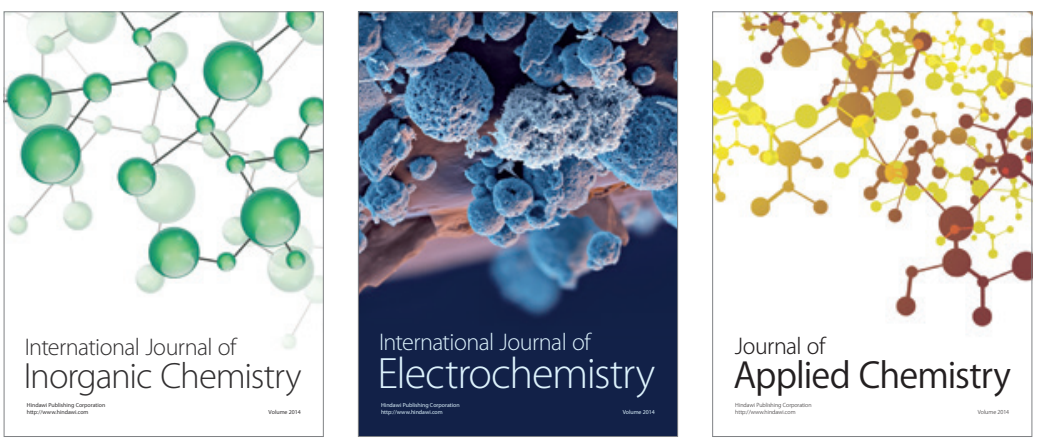

Journal of

Applied Chemistry
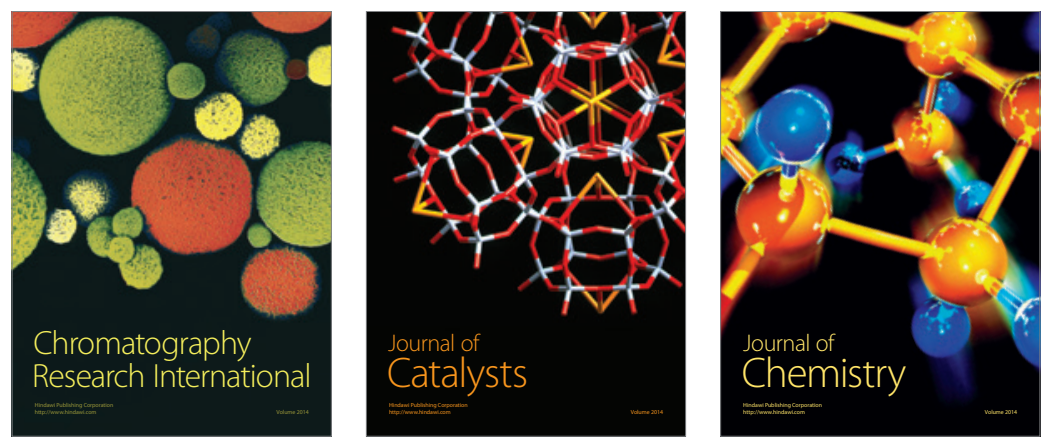
